\title{
Updates on the pathogenesis, epidemiology and diagnosis of Clostridium difficile infection
}

\begin{tabular}{|c|}
\hline 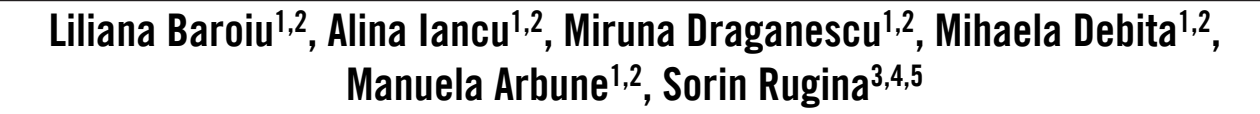 \\
\hline $\begin{array}{l}\text { 1“Dunarea de Jos" University of Galati, Faculty of Medicine and Pharmacy, Galati, Romania } \\
\qquad \begin{array}{c}\text { 2Clinical Hospital of Infectious Diseases, Galati, Romania } \\
{ }^{3} \text { Ovidius University of Constanta, Romania } \\
{ }^{4} \text { The Academy of Romanian Scientists } \\
{ }^{5} \text { The Academy of Medical Sciences }\end{array}\end{array}$ \\
\hline
\end{tabular}

\begin{abstract}
The infection with Clostridium difficile (CDI) is a cause of acute gastroenteritis (AGE), which is likely to severely develop into pseudomembranouse colitis (PMC), ileus and toxic megacolon. At the begining, CDI was considered a nosocomial infection, later proven to be community-acquired infections. The susceptibility for $\mathrm{CDI}$ is related to the alteration in intestinal microbiota after antibiotics or immunosuppressant treatments, postoperative disruption of mucosal barriers, trauma, tumor proliferation, ischemia or necrosis, as well as in other conditions caused by aging, alcoholism, diabetes, neoplasias, immunosuppression, angiopathies. Concern regarding the outbreak of new CDI-epidemics is still high, due to genetic and bacterial variability and spores resistance in outer environment. The diagnosis of CDI is a continuous challenge for clinicians, based on the correlation between clinical, epidemiological data and complex laboratory investigations.
\end{abstract}

Keywords: Clostridium difficile, toxines, infection control, diagnostic methods

\section{INTRODUCTION}

Clostridium difficile (CD) was first isolated in 1935 from the intestinal flora of the newborn infants, but the first documentation of pathogenicity for this bacteria dates back to 1978, when it was associated with a case of postantibiotic pseudomembranous colitis $(1,2)$. Clindamycin and $\beta$-lactamines with broad spectrum, were originally associated with diarrhea caused by $\mathrm{CD}$. Extensive use of fluoroquinolones over the past 20 years has contributed to the emergence of the hypervirulent PCR ribotype 027 strains and the increase in cases of Clostridium difficile infections (CDI) (3).

Medical interest for CDI increased after 2001, with the onset of PCR ribotype 027 , identified in the United States of America (USA) and Canada (4). The outbreak in the U.S. has been declared a public health issue in 2011, when the number of new cases has risen to 500,000/year, with 15,00030,000 deaths/year, supplementing the hospitalization expenses with over 4.8 bn. \$ (3). In the coming years, the ribotype 027 strain has spread across Europe, and then worldwide, based on the expected context of heavy and inappropriate consumption of antibiotics $(5,6)$.

Although a significant increase of CDI morbidity was experienced in hospitals from UK, the incidence of this infection has decreased by nearly $80 \%$ after 2006, due to a national programme for hospital infection control and transmission, but also by a strategy of restricting the use of antibiotics as a 
trigger factor in CDI-outburst (7). These principles have grounded a similar national program from Canada, where CDI-morbidity decreased between 2009-2015 by 38,5\% (8). Over 123000 cases are recorded in the European Union every year, representing the $8^{\text {th }}$-leading cause of health-care-associated infection, with a prevalence of 2.6 cases per 10,000 patients/day (5). In Romania, the first CDIcases of ribotype 027 were notified 2011 and the number of infections is continuous increasing. The prevalence of CDI evaluated by a multicentric national study revealed 5.2 cases per 10,000 patients/ day (9).

\section{PATHOGENESIS}

Clostridium difficile (CD) is a Gram positive bacillus, strictly anaerobic, sporulated, toxigenic, that colonizes the intestine, especially when a microbial imbalance occurs. The life cycle of CD is biphasic, involving a vegetative form which is responsible for producing pathogenic toxins and a dormant spore form, resistant to oxygen, dryness, low temperatures and most disinfectant agents. The resistance of CD spores in outer environment explains the persistence of this bacteria in nature and the difficulties encountered in infection prevention. Isolation of CD strains requires special media and anaerobic cultures from stool, blood or other tissues. The specific epithet "difficile" indicates the difficulty encountered during culturing and identification of this microbe (10).

Electron microscopy highlighted the main cellular changes induced by exposure to toxines of $\mathrm{CD}$ are the loss of cytoskeletal structure, cell rounding, interruption in cell-cell junctions and apoptosis. These changes alter the intestinal barrier and contribute to the accumulation of fluid in the intestinal lumen, associated with diarrhoeal disease $(11,12)$.

Virulence factors of CD are represented by the polysaccharidic capsule, adesines, enzymes and toxins. Nonpathogenic CD strains that do not produce toxin, do not produce infections. Toxins A and $\mathrm{B}$ are the most important factors of virulence and they are encoded by the genes tcdA and tcdB. There are 3 versions of $C D$ strains, as producing either toxin A (tcdA), toxin B (tcdB) or CD binary toxin CDT (13).

The role of these toxins is to regulate many biological processes, ensuring secretion, maintaining the cytoskeleton, cell adhesion, chemotaxis and stimulation of cytokines secretion, TNF, IL-1, IL-6 and IL-8 (14). Regarding the CDI pathogenesis, it is supposed that IL-8 plays a particular role, by neutrophil recruitment and activation, that were frequently found in the gut inflamed areas. The polymorphism of the gene IL-8 was associated with susceptibility to the recurrence of CDI (15). Currently, it has been proven that $\mathrm{CD}$ toxins act by glycosylating the Rho, Rac and Cdc42 GTP-ase proteins (16). Glycosylation prevents the activation of these proteins and their ability to regulate actin polymerization, altering the host-cells, with cytopathic, cytotoxic and immunologic response (17). However, this theory is not fully explaining the molecular mechanisms of these clostridial toxins, taking into account the complexity of Rho proteins. Inactivation of Rho proteins blocks NFkB, the transcription and TLRs secretion, induced by the inflammatory cytokines and chemokines, but which seem to be stimulated by CD toxins (17).

Genes' variability which encode A toxins (tcdA) and B toxins (tcdB) can be evaluated by toxinotyping, using methods of molecular genetics (PCRRFLP). There are 34 toxinotypes, characterized by the PaLoc modification ("pathogenicity locus"), made of the encoding regions tcd A and tcdB, alongside 3 regulatory genes: $t c d R, t c d C$ and $t c d E$ (18).

Deciphering the bacterial genome has allowed the identification of an increased number of ribotypes, resulting after point mutations, deletions or insertions of the "jumping genes" (transposons). They differ in terms of virulence, resistance to antibiotics and their interaction with the host. Toxitypes correlate with ribotypes. Around the world, the most common toxitypes are III (PCR ribotype 027), IV (PCR ribotype 023), V (PCR ribotype 078/126) and VIII (PCR ribotype 017) $(13,19)$.

\section{EPIDEMIOLOGY}

The epidemiology of CDI has changed in the last 20 years. Although the health-care associated cases continue to be majority, the number of community-acquired infections is increasing. Among community-acquired infections, $40 \%$ may require hospitalization, becoming sources of infections in the hospital (20).

There are various sources of CDI, as the small intestine of sick or healthy subjects, the small intes- 
tine of various species of animals, the soil, water, or contaminated objects. The transmission of $\mathrm{CD}$ is by the digestive route, directly or indirectly by contaminated objects. Infections occurred in hospitals are mainly transmitted between patients and between patients and medical staff, but also after contact with medical equipments, medical protective clothing, healthcare furniture or contaminated floors (20,21). Community-acquired infections may be associated with various exposures in outer environment (dust, navigable waters, sewage, irrigation ditches), domestic environment (kitchen surfaces, refrigerators) and contact with animals or foods of animal origin $(22,23)$. Clostridium difficile, especially ribotype 078 and ribotype 027, which was isolated in cattles, pigs or domesticated poultries, but also pets and wild animals (22).

Receptiveness to CDI is induced by the imbalance of gut microbiota through the occurrence of some "gaps" in microbial composition. The impact of $\mathrm{CD}$ varies depending on age. Although more than $50 \%$ of infants are colonized by $\mathrm{CD}$, the infection is rarely diagnosed at this age because most cases are associated with nontoxigenic strains (24). A higher risk of colonization was observed to artificially fed infants than to breast-fed ones (25). Intestinal carriage of $\mathrm{CD}$ in healthy adults is much more rare, in $3-5 \%$ of subjects $(26,27)$. Most cases of symptomatic infections are diagnosed after the age of 65 , especially in patients who underwent surgical procedures, oncological diseases and chronic kidney disease (28).

The risk of community-acquired infections with $\mathrm{CD}$ is higher in young patients, women who take care of infants, people who receive proton-pump inhibitors (PPIs) or antibiotics with broad spectrum, people living in the neighbourhood of farms or livestock farmers (20).

\section{DIAGNOSIS}

The difficulty of CDI diagnosis is influenced by the increased rate of asymptomatic colonization in hospitalized patients, that was reported between $7 \%$ and $26 \%$ (29). Therefore, the evaluation of clinical criteria consistent with case definitions is essential for the correct interpretation of the investigations carried out for the diagnosis of CDI. The clinical definition proposed by Dubberke and his collaborators refers to more than 3 bowel movements within 24 hours, low consistency stools with 5-7 points according Bristol Stool Form Scale and abdominal pain or cramps (30). Currently, the case definition recommended by practical guidelines comprises three clinical variants, diarrhoea, megacolon or severe ileus and a positive laboratory test or a pseudomembranous aspect revealed at the endoscopic or histological examination $(3,5,10)$.

The diagnosis of CDI case can be new or recurring, if it follows another episode, confirmed in the last 2-4 weeks (3). Refered to the relation of hospital admission and symptoms onset, the CDI are classified as nosocomial, from 48 hours after admission, up to 28 days after discharge or community-acquired, if occure over 8 weeks after discharge. Indeterminate CDI case is characterized by the onset of the symptoms between 4 and 8 weeks after discharge $(5,28,29)$. Dehydration syndrome, even hypovolemic shock, sepsis and acute abdomen are the complications of CDI. Risk factors for CDI complications are advanced age, renal insufficiency, leukocytosis and comorbidities. The risk of death was associated with age, comorbidities, hypoalbuminemia, leukocytosis, kidney faillure and the ribotype 027 . The ATLAS Score quantifies the risk of death, taking into consideration the age, the presence of fever, the severity of leukocytosis and hypoalbuminemia, as well as the use of systemic antibiotics (31).

Laboratory diagnosis is required only in cases with risk factors and clinical criteria, after excluding drug-induced diarrhoea or other causes of diarrhoeal disease. It is not recommended to test consistent stool for CD, except the paralytic ileus case (32). The diagnosis methods indicated for CDI are toxigenic culture (TC) cell cytotoxicity neutralization assay (CCNA), enzyme immunoassay (EIA) for glutamate-dehydrogenase (GDH), tcdA and tcdB and nucleic acid amplification test (NAAT). However, none of these methods is considered the reference standard. Over the past 30 years, CDI diagnosis was based on TC combined with NAAT, but these methods are laborious and time-consuming. Other disadvantages are the difficult interpretation and low reproducibility of the results, due to low specificity of the toxigenic culture, proven sensitivity of the assay for neutralizing only for toxin B (33). 
Anaerobiosis culture is achieved on selective agar media with cicloserine cefoxitin-fructose, after prior decontamination of the stool sample by treating with heat or alcohol. After a few days incubation at temperatures of $20-25^{\circ} \mathrm{C}$, there may grow yellowish, planar colonies with irregular margins and the appearance of frosted glass. Culture sensitivity may be increased by up to $100 \%$ by using ChromoID medium, agar enriched with sodium taurocholate, egg yolk agar, trypticase soy and sheep blood agar (34) (Figures 1, 2).

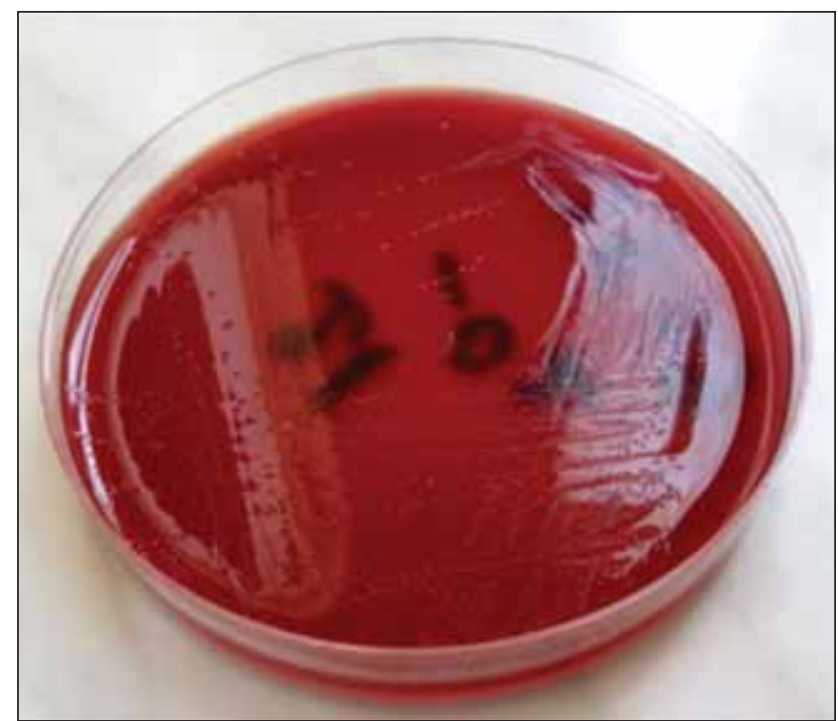

FIGURE 1. Culture of Clostridium difficile on agar-blood medium, with non-haemolytic colonies, matte surface and diameter of 2-4 $\mathrm{mm}$

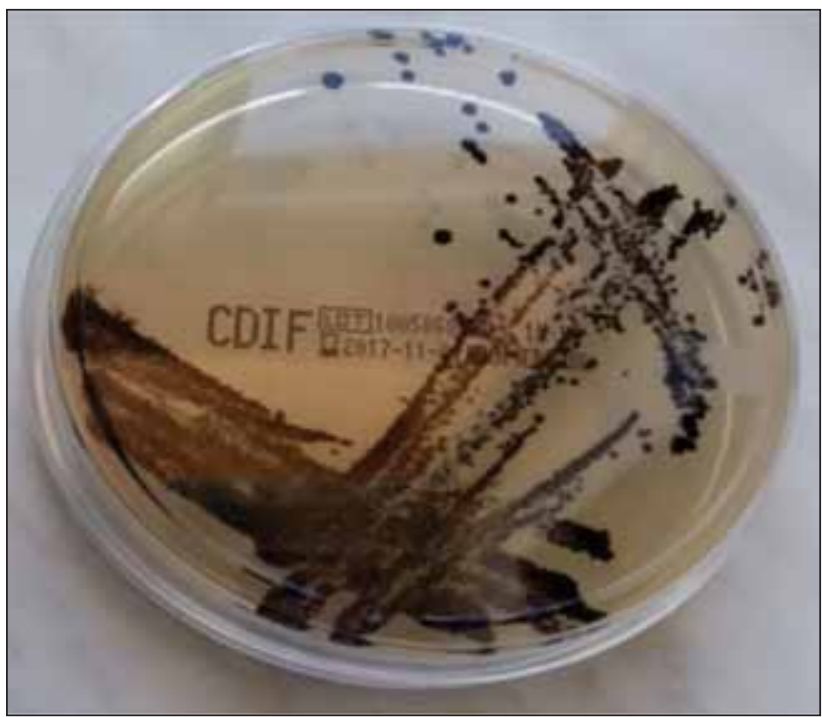

FIGURE 2. Culture of Clostridium difficile on chromogenchromium medium C. difficile agar (bioMérieux) - black colonies

Presumption tests are run on suspected colonies: morphology of the colonies, the appearance in
Gram staining (Figure 3) and biochemical characteristics, with positivity for indole and L-prolinenaphthalimide tests. Positive presumption tests require confirmation by RapID ANA systems (rapid identification system of anaerobic isolates) or MALDI-TOF (matrix-assisted laser desorption/ ionization- time of flight) (35). The identified strains of CD can be nontoxigenic (20-25\%). After identifying strains of $\mathrm{CD}$, it is necessary to assess the pathogenic potential using a toxigenesis test, CCNA, EIA or NAAT (3).

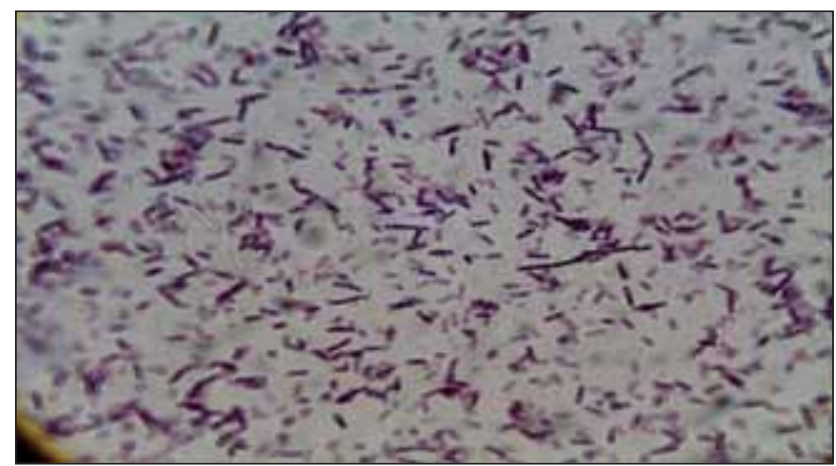

FIGURE 3. Gram staining smear - Clostridium difficile culture - optical microscopy

Cell cytotoxicity neutralization assay detects $\mathrm{CD}$ toxin by identifying cell the cytopathic effect on McCoy MRC-5 or Vero cell cultures. In the presence of the toxin, cells present a reversible cytopathic effect after neutralization with antitoxin (34).

Commercial EIA tests for detecting the toxins $\mathrm{A} / \mathrm{B}$ and GDH are fast and have relatively low costs, but sensitivity and specificity are low and require to be used combined. The EIA-GDH test is recommended for screening, due to good sensitivity, although may present cross reactions with the enzymes of other species of clostridia and does not distinguish toxigenic strains to the nontoxigenic ones (36).

Using NAAT identifies the genes which encode tcdA and tcdB and/or the binary toxin, by PCR (polymerase chain reaction) or LAMP (loop-mediated isothermal amplification). The advantages of NAAT are speed, low complexity and high accuracy, while presents disadvantage in detecting nontoxigenic strains detection, with a high rate of false-positive results (37).

European Society of Clinical Microbiology and Infectious Diseases (ESCMID) recommends using 
a two-step diagnosis algorithm, beginning with a GDH-EIA or NAAT test, followed by an EIA test in order to confirm the toxin (38).

Intestinal inflammation have been associated with CD toxins, given by the presence of biomarkers in stool samples, lactoferrin, myeloperoxidase, cytokines and calprotectin. These biomarkers, may be indicators of severity, easily determinable, but not specific to CDI and they are not recommended by the majority of practical guidelines (34).

Endoscopic methods, flexible sigmoidoscopy and colonoscopy are no longer due to fragile nature of the lining of the colon and risk of wall perforation (39). Colonoscopy and anatomopathological examination of surgical or necrotic specimens, can establish the diagnosis of CDI by revealing typical pseudomembranes, with yellowish deposits and pseudomembranes (Figure 4). Microscopically, microscopic examination describes the damaged epithelium with focal necrosis areas, neutrophilic infiltrate of the mucosa, and fragmented clumps of leukocytes, fibrin, mucus and cell detrituses $(10,16)$.

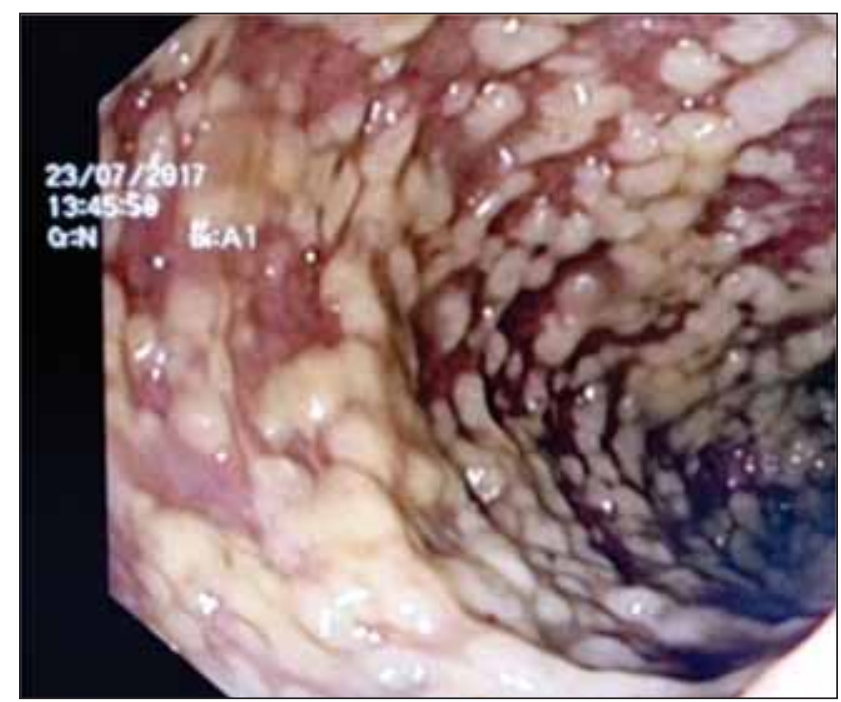

FIGURE 4. Colonoscopy image - Clostridium difficile infection, mucosal inflammation with yellow-coated deposits of 1-4 mm

Imaging methods are recommended for the diagnosis of CDI, when complications or other intraabdominal pathologies are suspected. The most common aspect highlighted in abdominal $\mathrm{CT}$ is bowel wall thickening, to which ascites and bowel irregularity of the wall can associate (Figures 5,6). The abdominal $\mathrm{x}$-ray is indicated for early diagnosis of toxic megacolon, especially in patients presenting sepsis criteria $(10,36)$.

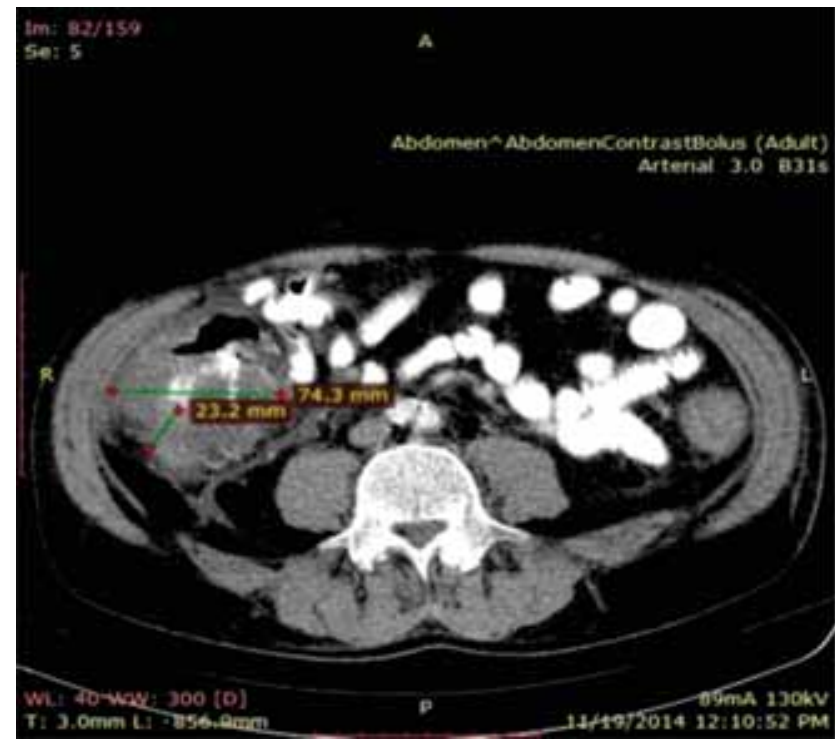

FIGURE 5. Abdominal CT-scan - Ascending colon - wall thickness of 23,2 $\mathrm{mm}$, transversal diameter of $74,3 \mathrm{~mm}$

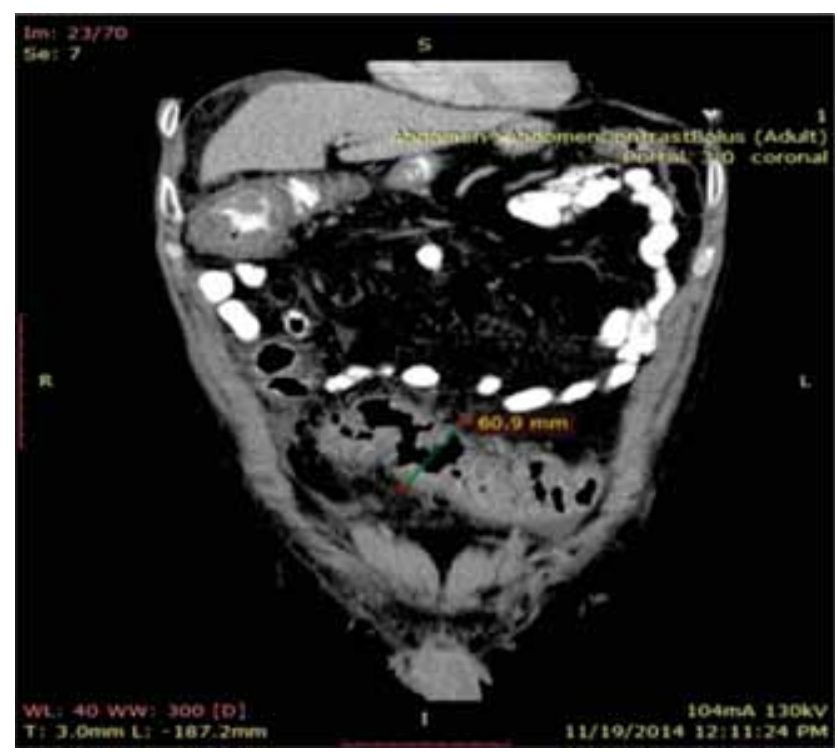

FIGURE 6. Abdominal CT Scan Image - Sigmoid column diameter of $60.9 \mathrm{~mm}$ (coronal section) (41)

Current studies are focused on the development of ultrasensitive ELISA tests (enzyme-linked immunosorbent assays) allowing quantitative detection of the two toxins, tcdA and tcdB. Another research line is the metabolomic analysis from urine and stool samples. Based on the assumption that the microbiota of healthy subjects with CDI is altered due to antibiotics, metabolomic analysis has revealed that cholesterol and coprostanol levels from stool samples are different from the ones in healthy subjects. Among the 53 studied metabolites in urine samples, choline submitted a significant difference in CDI comparative with healthy subjects (40). 
In conclusion, CDI-epidemic continues to spread worldwide, although national prevention programmes have succeeded in controlling nosocomial infections in some countries of the world. CDI-pathogenesis is complex and dynamic, with the possibility of emmergence in new virulent ri-

\section{REFERENCES}

1. George WL, Sutter VL, Goldstein EJ et al. Aetiology of antimicrobial - agent associated colitis. Lancet. 1978;1:802-803.

2. Bartlett JG, Moon N, Chang TW et al. Role of Clostridium difficile in antibiotic-associated pseudomembranous colitis. Gastroenterology. 1978;75:778-782.

3. McDonald C, Gerding D, Johnson S, Bakken J, Carroll K, Coffin S, Dubberke E, Garey K, Gould C, Kelly C, Loo V, Shaklee SJ, Sandora T, Wilcox M. Clinical Practice Guidelines for Clostridium difficile Infection in Adults and Children: 2017 Update by the Infectious Diseases Society of America (IDSA) and Society for Healthcare Epidemiology of America (SHEA). Clin Infect Dis. 2018;66(7):e1-e48.

4. He M, Sebaihia M, Lawley TD, Stabler RA et al. Evolutionary dynamics of Clostridium difficile over short and long time scales. Proc Natl Acad Sci U S A. 2010;107(16):7527-32.

5. European Centre for Disease Prevention and Control. Healthcareassociated infections: Clostridium difficile infections. In: ECDC. Annual epidemiological report for 2016, Stockholm: ECDC, 2018. https://ecdc.europa.eu/sites/portal/files/documents/AER_for_2016-Cdifficile_0.pdf

6. Rafila A, Indra A, Popescu GA et al. Occurrence of Clostridium difficile infections due to PCR ribotype 027 in Bucharest, Romania, 2011 to 2012, The Journal of Infection in Developing Countries. 2014; 8(6):694-698.

7. Dingle KE, Didelot X, Quan TP et al. Effects of control interventions on Clostridium difficile infection in England: An observational study. Lancet Infect Dis. 2017;17(4):411-421.

8. Katz KC, Golding GR, Choi KB et al. The evolving epidemiology of Clostridium difficile infection in Canadian hospitals during a postepidemic period (2009-2015). CMAJ, 2018;190 (25):E758-E765.

9. Popescu GA, Serban R, Pistol A et al. The Recent Emergence of Clostridium difficile Infection in Romanian Hospitals is Associated with a High Prevalence of Polymerase Chain Reaction Ribotype 027. Balkan Med J. 2018;35(2):191-195.

10. Thielman NM. Antibiotic-associated colitis. In: Mandell GL, Bennett $\mathrm{JE}$, Dolin R, editors. Mandell, Douglas, and Bennett's Principles and Practice of Infectious Diseases. 5th ed.Vol 1. Edinburgh: Churchill Livingstone; 2000. p. 1111-26.

11. Feltis BA, Wiesner SM, Kim AS, Erlandsen SL, Lyerly DL, Wilkins TD, Wells CL. Clostridium difficile toxins A and B can alter epithelial permeability and promote bacterial paracellular migration through HT-29 enterocytes. Shock. 2000; 14:629-634.

12. Johal SS, Solomon K, Dodson S, Borriello SP, Mahida YR. Differential effects of varying concentrations of Clostridium difficile toxin A on epithelial barrier function and expression of cytokines. J. Infect. Dis. 2004; 189:2110-2119.

13. Kuehne SA, Collery MM, Kelly ML, Cartman ST, Cockayne A, Minton NP. Importance of toxin A, toxin B, and CDT in virulence of an epidemic Clostridium difficile strain, The Journal of Infectious Diseases. 2014; 209:83-86.

14. Savidge TC, Pan WH, Newman P, O'Brien M, Anton PM, Pothoulakis C. Clostridium difficile toxin $B$ is an inflammatory enterotoxin in human intestine. Gastroenterology. 2003; 125:413-420.

15. Jiang ZD, Dupont HL, Garey K, Price M, Graham G, Okhuysen P, Dao-Tran T, Larocco M. A common polymorphism in the interleukin 8 gene promoter is associated with Clostridium difficile diarrhea. Am. J. Gastroenterol. 2006; 101:1112-1116.

16. Vedantam G, Clark A, Chu M et al. Clostridium difficile infection. Gut Microbes 2012;3:121-34. botypes. CDI-diagnosis remains a challenge related to the difficulties of bacterial identification and interpretation of laboratory results, which always require correlation with clinical and epidemiological data.

Conflict of interest: none declared Financial support: none declared

17. Chen S, Sun C, Wang H, Wang J. The Role of Rho GTPases in Toxicity of Clostridium difficile Toxins. Toxins (Basel). 2015; 7(12):5254-67. Published 2015 Dec 2. doi:10.3390/toxins7124874.

18. Rupnik M, Janezic S. 2016. An update on Clostridium difficile toxinotyping. J Clin Microbiol 54:13-18.

19. Loo VG, Poirier L, Miller MA, Oughton M, Libman MD, Michaud S et al. A predominantly clonal multi-institutional outbreak of Clostridium difficile-associated diarrhea with high morbidity and mortality, The New England J. of Medicine. 2005; 353:2442-2449.

20. Ofori, E; Ramai, D; Dhawan, M; Mustafa, F; Gasperino, J; Reddy, M. Community-acquired Clostridium difficile: Epidemiology, ribotype, risk factors, hospital and intensive care unit outcomes, and current and emerging therapies. The Journal of hospital infection, 2018; 99(4):436-442.

21. Longtin $Y$, Paquet-Bolduc B, Gilca $R$ et al. Effect of detecting and isolating Clostridium difficilecarriers at hospital admission on the incidence of $\mathrm{c}$ difficile infections: A quasi-experimental controlled study, JAMA Intern Med, 2016; 176(6):796-804.

22. Brown AWW, Wilson RB. Clostridium difficile colitis and zoonotic origins-a narrative review. Gastroenterol Rep (Oxf). 2018;6(3):157-166.

23. Rabold D, Espelage W, Abu Sin M et al. The zoonotic potential of Clostridium difficile from small companion animals and their owners. PLoS One. 2018;13(2):e0193411. doi:10.1371/journal.pone.0193411

24. Jangi S, Lamont JT. Asymptomatic colonization by Clostridium difficile in infants: implications for disease in later life. $J$ Pediatr Gastr. Nutr 2010; 51:2-7.

25. Penders J, Stobberingh EE, van den Brandt PA, van Ree R, Thijs C. Toxigenic and non-toxigenic Clostridium difficile: determinants of intestinal colonisation and role in childhood atopic manifestations. Gut 2008; 57:1025-6.

26. Blixt T, Gradel KO, Homann C et al. Asymptomatic Carriers Contribute to Nosocomial Clostridium difficile Infection: A Cohort Study of 4508 Patients. Gastroenterology, 2017:152 (5):1031-1041. e2. doi.org/10.1053/j.gastro.2016.12.035.

27. Lanzas C, Dubberke ER. Effectiveness of screening hospital admissions to detect asymptomatic carriers of Clostridium difficile: a modeling evaluation. Infect Control Hosp Epidemiol 2014; 35:1043-50.

28. Dorp SM, Kinross P, Gastmeier P, Behnke M, Kola A, Delmee M et al. Standardised surveillance of Clostridium difficile infection in European acute care hospitals: AS pilot study, 2013. Euro Surveill. 2016;21(29).

29. Cohen, SH, Gerding DN, Johnson S, Kelly CP, Loo VG, McDonald LC, Wilcox MH (2010). Clinical practice guidelines for Clostridium difficile infection in adults: 2010 update by the Society for Healthcare Epidemiology of America (SHEA) and the Infectious Diseases Society of America (IDSA). Infection Control and Hospital Epidemiology, 31(5), 431-455. https://doi.org/10.1086/651706.

30. Dubberke ER, Han Z, Bobo L et al. Impact of clinical symptoms on interpretation of diagnostic assays for Clostridium difficile infections. J Clin Microbiol 2011; 49:2887-93.

31. Debast S B, Bauer M P and Kuijper E J on behalf of the Committee European Society of Clinical Microbiology and Infectious Diseases: Update of the Treatment Guidance Document for Clostridium difficile Infection. Clinical Microbiology and Infection, 2014;20(S2):1-26. doi. org/10.1111/1469-0691.12418.

32. Crobach MJT, Planche T, Eckert C, Barbut F, Terveer EM, Dekkers OM, Wilcox MH, Kuijper EJ. European Society of Clinical Microbio- 
logy and Infectious Diseases: Update of the diagnostic guidance document for Clostridium difficile infection. Clin. Microbiol. Infect. 2016;22(Suppl. 4):S63-S81. doi: 10.1016/j.cmi.2016.03.010.

33. Murad YM, Perez J, Ybazeta $G$ et al. False Negative Results in Clostridium difficile Testing. BMC Infect Dis. 2016;16(1):430. doi:10.1186/s12879-016-1741-6.

34. Martínez-Meléndez A, Camacho-Ortiz A, Morfin-Otero R et al. Current knowledge on the laboratory diagnosis of Clostridium difficile infection. World J Gastroenterol 2017;23:1552.

35. Li R, Xiao D, Yang J, et al. Identification and Characterization of Clostridium difficile Sequence Type 37 Genotype by Matrix-Assisted Laser Desorption lonization-Time of Flight Mass Spectrometry. J Clin Microbiol. 2018;56(5):e01990-17. doi:10.1128/JCM.01990-17.

36. Peng Z, Ling L, Stratton CW et al. Advances in the diagnosis and treatment of Clostridium difficile infections. Emerg Microbes Infect. 2018;7(1):15. doi:10.1038/s41426-017-0019-4.
37. Kendrick K. Laboratory diagnosis of Clostridium difficile infection. J Lab Precis Med 2018;3:20. doi: 10.21037/jlpm.2018.02.05.

38. European Centre for Disease Prevention and Control. Laboratory procedures for diagnosis and typing of human Clostridium difficile infection. Stockholm: ECDC; 2018.

39. Lübbert C, John E, von Müller L. Clostridium difficile Infection Guideline-Based Diagnosis and Treatment. Dtsch Arztebl Int 2014;111:72331.

40. Kao D, Ismond KP, Tso $V$ et al. Urine-based metabolomic analysis of patients with Clostridium difficile infection: a pilot study. Metabolomics 2016;12:135.

41. Draganescu M, Baroiu L, lancu A, Arbune M, Dumitru I. M, Rugina S. Clostridium difficile Infection Complicated By Toxic Megacolon In Immunocompetent Patient. ARS Medica Tomitana. 2017; 1(23): 39-46. 